\title{
Obtención de hidrogeles de quitosano/Rosmarinus officinalis modificados con plasma
}

\author{
Obtaining chitosan hydrogels/Rosmarinus officinalis modified with plasma

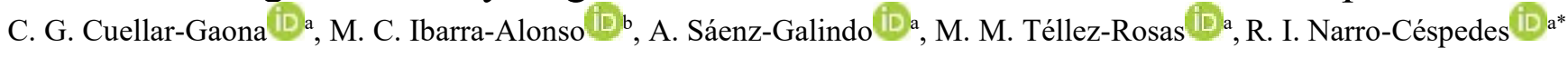 \\ ${ }^{a}$ Facultad de Ciencias Químicas, Universidad Autónoma de Coahuila, Blvd. Venustiano Carranza S/n, Col. República Oriente C.P. 25280, Saltillo, Coahuila, México. \\ ${ }^{b}$ CONACYT. Facultad de Ciencias Químicas, Universidad Autónoma de Coahuila, Blvd. Venustiano Carranza S/n, Col. República Oriente C.P. 25280, Saltillo, \\ Coahuila, México.
}

\begin{abstract}
Resumen
Los hidrogeles son redes tridimensionales con gran contenido de agua, tienen diferentes propiedades de acuerdo al material con el que se obtengan. Estos materiales a base de polímeros naturales presentan propiedades de biocompatibilidad, biodegradabilidad, entre otras. Sin embargo, estas propiedades pueden mejorarse utilizando tecnología de plasma, el cual es un procedimiento con la capacidad de modificar superficies para funcionalizar, esterilizar o polimerizar un material. En el presente trabajo se obtuvieron hidrogeles a base de quitosano con diferentes concentraciones de ácido acético glacial, se caracterizaron por las técnicas FTIR-ATR, DSC y TGA. Para la evaluación de la biocompatibilidad, se realizó un ensayo hemolítico, y se comparó un blanco, contra un hidrogel con extracto de romero y otro hidrogel con extracto de romero y modificado con plasma. Los resultados obtenidos demostraron que la tecnología de plasma es una herramienta innovadora para mejorar propiedades biológicas al modificar superficies.
\end{abstract}

Palabras Clave:

Hidrogel, Quitosano, Hemólisis, Plasma, Biocompatibilidad.

\begin{abstract}
Hydrogels are three-dimensional networks with high water content, hydrogels have different properties according to the material with which they are obtained. These materials based on natural polymers have good biocompatibility and biodegradability properties, among others. However, these properties can be improved using plasma technology, which is a procedure with the ability to modify surfaces to functionalize, sterilize or polymerize a material. In the present work, hydrogels based on chitosan with different concentrations of glacial acetic acid were obtained, they were characterized by FTIR-ATR, DSC and TGA techniques. For the evaluation of biocompatibility, a hemolytic test was performed, and a blank was compared against a hydrogel with rosemary extract and another hydrogel with rosemary extract and modified with plasma. The results obtained showed that plasma technology is an innovative tool to improve biological properties by modifying surfaces.
\end{abstract}

Keywords:

Hydrogel, Chitosan, Hemolysis, Plasma, Biocompatibility.

\section{Introducción}

Los hidrogeles son materiales blandos formados por redes tridimensionales que absorben un alto contenido de agua manteniendo su estructura. Tienen un gran parecido a los tejidos biológicos (Liu, Ji et al. 2020; Wei, Ma et al. 2021). Son elásticos, biocompatibles y biodegradables, con capacidad para responder a estímulos externos (Maiz-Fernández, Guaresti et al. 2020). Los hidrogeles sirven como apósitos para heridas para mantener un ambiente húmedo en la herida y como vehículo para entrega de fármacos para administrar agentes terapéuticos como antibióticos, factores de crecimiento, células madre, etc (Tran, Le Thi et al.
2020). Los hidrogeles pueden obtenerse a partir de materiales de diversas fuentes. Los biopolímeros representan un área de gran interés en los últimos años debido a su origen natural, su capacidad de renovación y sus propiedades ecológicas (Ahmed, Mohamed et al. 2020). Entre los biopolímeros más utilizados se encuentran el ácido hialurónico, colágeno, gelatina, quitosano, alginato, celulosa (Dhand, Galarraga et al. 2020), almidón, ácido algínico, entre otro (Forouzandehdel, Forouzandehdel et al. 2020). Una ventaja del uso de biopolímeros es que tienen propiedades interesantes como la bioactividad, biodegradabilidad y biocompatibilidad, la desventaja es que sus propiedades

*Autor para la correspondencia: rinarro@uadec.edu.mx

Correo electrónico: gabriela_cuellargaona@uadec.edu.mx (Claudia Gabriela Cuellar-Gaona), ibarra.cristina@uadec.edu.mx (María Cristina Ibarra-Alonso), aidesaenz@uadec.edu.mx (Aide Sáenz-Galindo),mauratellez@uadec.edu.mx (María Maura Téllez-Rosas), rinarro@uadec.edu.mx (Rosa Idalia Narro-Céspedes 
mecánicas generalmente son más débiles (Dhand, Galarraga et al. 2020).

El quitosano es de los polisacáridos más utilizados como biomateriales, comprende copolímeros de unidades $\mathrm{N}$ glucosamina y $\mathrm{N}$-acetilglucosamina unidas por enlaces $\beta-1,4-$ glicosídicos. Es un material muy reactivo debido a la presencia de grupos amino e hidroxilo en su estructura (Ahmed, Mohamed et al. 2020). Tiene propiedades antioxidantes, biocompatibles, biodegradables, baja toxicidad, antimicrobianas, y hemostáticas (Tran, Le Thi et al. 2020; Rezaei, Sharifianjazi et al. 2021). El hidrogel a base de quitosano tiene propiedades de gel mejor controladas, incluida la resistencia mecánica $(\mathrm{Lu}$, Huang et al. 2020). La desventaja del quitosano es que no es soluble en agua, por lo que requiere un medio ácido con un $\mathrm{pH}$ inferior a 6 , para que pueda disolverse como consecuencia de la protonación de los grupos amino de las cadenas de quitosano (Maiz-Fernández, Guaresti et al. 2020). El romero (Rosmarinus officinalis) es un arbusto de hoja perenne que se cultiva en todo el mundo (Zhong, Wang et al. 2020). En la medicina se ha utilizado desde la antigüedad para atender diversas afecciones ya que es antioxidante, antimicrobiano, antiespasmódico, antirreumático, diurético, antidiabético, antiepiléptico, antidepresivo, hepatoprotector, actividades contra el cáncer y para mejorar la disfunción de la memoria (Karadağ, Demirci et al. 2019). Estas actividades biológicas dependen de su composición química contiene diferentes quimiotipos de $\alpha$-pineno, 1-8 cineol, alcanfor, borneol, acetato de bornilo (Elmi, Prosperi et al. 2019; Ben Abada, Haouel Hamdi et al. 2020) canfeno, catequina y grandes cantidades de hidrocarburos (Loto and Loto 2019). Estudios han demostrado que las fracciones antibacterianas más potentes del extracto de romero, preparadas con disolventes orgánicos mediante ultrasonido, impregnación u otros métodos, contienen principalmente compuestos no volátiles, como el ácido carnósico, carnosol, ácido rosmarínico, romanol, rosmaridifenol, isorosmanol y epirosmanol (Zhong, Wang et al. 2020).

El uso de tecnología de plasma es una herramienta novedosa que permite modificar superficies para que estas superficies cuenten con características diferentes, tiene la capacidad de esterilización y limpieza del material, formación de recubrimientos que logran que la superficie pase de ser hidrofílica a hidrófoba, funcionalización y activación mediante la inserción de radicales activos. El objetivo del presente trabajo fue obtener hidrogeles de quitosano cargados con extracto de romero para ser modificados con tecnología de plasma para evaluar su biocompatibilidad. Esta propiedad se evaluó por medio de la prueba de hemólisis, la cual mide el porcentaje de liberación de hemoglobina al momento de la destrucción de los eritrocitos en contacto con el hidrogel.

\section{Materiales y métodos}

Los reactivos para la síntesis de hidrogel se adquirieron en Sigma Aldrich y se utilizaron sin ningún tratamiento adicional, quitosano de alto peso molecular (300 KDa), ácido acético glacial (grado reactivo 99.98\%) e hidróxido de sodio (grado reactivo 98\%).

El extracto de romero se obtuvo por radiación ultrasónica, utilizando acetato de etilo para la extracción.

Para la prueba de hemólisis la solución Alsever se preparó de la siguiente manera: $2.05 \%$ de dextrosa, $0.8 \%$ de citrato de sodio, $0.055 \%$ de ácido cítrico y $0.42 \%$ de cloruro de sodio.

\subsection{Síntesis del hidrogel de quitosano}

Se prepararon hidrogeles utilizando diferentes concentraciones de ácido acético glacial. Se disolvió $3 \%$ de quitosano para la síntesis de cada hidrogel, en ácido acético glacial con las siguientes concentraciones: $0.5,1$ y $2 \%$ (HQ 0.5 , HQ 1 y HQ 2, respectivamente). Las soluciones se mantuvieron en agitación constante. El pH de las soluciones fue ajustado a 7 con $\mathrm{NaOH} 1$ M para obtener los hidrogeles. Posteriormente, se secaron en una estufa a $45^{\circ} \mathrm{C}$ durante 3 días para llevarse a cabo la caracterización.

\subsection{Caracterización de los hidrogeles de quitosano}

Los hidrogeles se caracterizaron mediante las técnicas de espectroscopía infrarroja (FTIR-ATR), en un equipo Thermo Scientific Nicolet iS10 en un rango de 600 a $4000 \mathrm{~cm}^{-1}$ con 100 escaneos y una resolución de $0.4 \mathrm{~cm}^{-1}$; calorimetría diferencial de barrido (DSC) en un calorímetro Shimadzu DSC-60. 54. Inicialmente, se realizó un calentamiento a $20^{\circ} \mathrm{C} / \mathrm{min}$ de 30 a 200 ${ }^{\circ} \mathrm{C}$, y se mantuvo la temperatura a $200{ }^{\circ} \mathrm{C}$ durante 1 minuto con el fin de borrar el historial térmico de las muestras. Posteriormente las muestras se enfriaron a $20{ }^{\circ} \mathrm{C} / \mathrm{min}$ hasta $30{ }^{\circ} \mathrm{C}$; y análisis termogravimétrico (TGA) en un equipo Perkin Elmer TGA 4000. Los análisis se desarrollaron en una atmósfera inerte de $\mathrm{N}_{2}$, con una velocidad de calentamiento de $20{ }^{\circ} \mathrm{C} / \mathrm{min}$, desde 30 a $800{ }^{\circ} \mathrm{C}$.

Después de la caracterización los hidrogeles fueron cargados con extracto de romero, para esto se sumergieron en $2 \mathrm{ml}$ de acetato de etilo que contenía $0.05 \mathrm{mg} / \mathrm{mL}$ de extracto de romero, durante $24 \mathrm{~h}$. Finalmente, se llevaron a la estufa a una temperatura de $45^{\circ} \mathrm{C}$ durante 3 día, para eliminar el solvente. La superficie del hidrogel se irradió con plasma frío, para llevar a cabo la modificación. Las condiciones en las que operó el reactor de plasma fueron las siguientes: una radiofrecuencia de $13.56 \mathrm{MHz}$, empleando aire como recurso, bajo una presión de $4.5 \times 10^{-1} \mathrm{mbar}$, durante 30 minutos.

\subsection{Prueba de hemólisis}

Para la prueba de hemólisis se evaluó el hidrogel a base de quitosano disuelto en $2 \%$ de ácido acético glacial, ya que fue el hidrogel más maleable para trabajar; fue cargado con extracto de romero (HQR), y se comparó con el blanco que no tenía carga de romero y contra el hidrogel de quitosano también cargado con romero y con tratamiento adicional de plasma (HQRM). La prueba de hemólisis se realizó con sangre humana recién obtenida de donantes voluntarios no fumadores, y se lavó tres veces con solución Alsever. Las muestras se prepararon en concentraciones de $1,2.5$ y $5 \mathrm{mg} / \mathrm{mL}$ del hidrogel previamente secado (Los hidrogeles se secaron en una estufa a $45^{\circ} \mathrm{C}$ durante 3 días, ya que la presencia de agua o algún solvente puede provocar la lisis de los eritrocitos y como consecuencia la liberación de hemoglobina). Las muestras se incubaron a $36.5^{\circ} \mathrm{C}$ en agitación constante durante $1 \mathrm{~h}$. la solución Alsever y el agua desionizada se utilizaron como controles negativo y positivo respectivamente. Las muestras se centrifugaron a $3000 \mathrm{rpm}$ durante 4 minutos y la hemoglobina libre en el sobrenadante se midió espectrofotométricamente por UV a $415 \mathrm{~nm}$.

\section{Resultados y discusiones}

La Figura 1 muestra los resultados de los espectros FTIR-ATR. Alrededor de los $3350 \mathrm{~cm}^{-1}$ se presenta una banda correspondiente a la tensión simétrica del $\mathrm{O}-\mathrm{H}$ que se atribuye a la presencia del quitosano, las bandas de 2900 a $2800 \mathrm{~cm}^{-1}$ se atribuyen al estiramiento de $\mathrm{CH}$; en $1650 \mathrm{~cm}^{-1}$ se aprecia una banda que representa al carbonilo $\mathrm{C}=\mathrm{O}$ del grupo acetilo y la banda a 1050 $\mathrm{cm}^{-1}$ corresponde al enlace $\mathrm{C}-\mathrm{O}-\mathrm{C}$ presente en la estructura del quitosano (Ahmed, Mohamed et al. 2020). En $1550 \mathrm{~cm}^{-1}$ se encuentra la vibración de flexión de la amida II, que se superpone con la flexión del grupo amino (Lopez-Carrizales, Mendoza- 
Mendoza et al. 2020). El hidrogel con menos concentración de ácido acético glacial (HQ 0.5), y una concentración de quitosano del $3 \%$, resultó ser el hidrogel más rígido en su estructura física, en el espectro se observa que es el que presenta una banda menos intensa de los grupos hidroxilo, por el contrario, el hidrogel con una concentración de quitosano del 3\% y una concentración de ácido del 1\% (HQ 1), aun fue rígido, mientras que el hidrogel HQ 2 fue el más maleable, por lo tanto se infiere que su estabilidad física depende en gran medida de la concentración del ácido acético glacial en el que se disuelva el quitosano, sin embargo, no hay reportes en la literatura de este comportamiento.

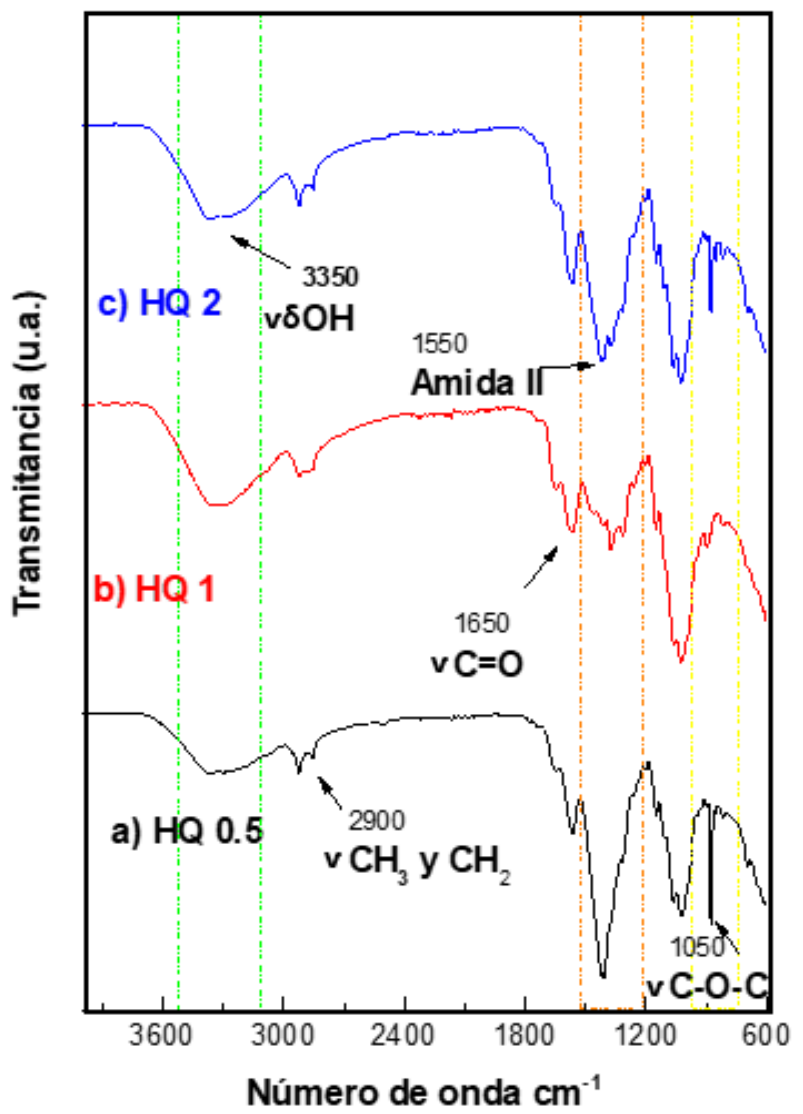

Figura 1. Espectro de FTIR-ATR de los hidrogeles de quitosano con diferentes concentraciones del ácido acético glacial.

Las propiedades térmicas de las diferentes relaciones de hidrogeles de quitosano, se evaluaron mediante la técnica DSC. El quitosano, como la quitina y la celulosa no exhiben claramente la transición vítrea (Tg) (Dong, Ruan et al. 2004; Mucha and Pawlak 2005), esto está relacionado a la ineficiente eliminación de agua, la técnica de preparación de la muestra a evaluar, la sensibilidad del equipo, así como la técnica de medición. La estimación de la $\mathrm{Tg}$ se fundamenta, con numerosos estudios de este biopolímero, donde no se ha establecido un valor determinado, sino un promedio que va desde los $100^{\circ} \mathrm{C}$ hasta los $160{ }^{\circ} \mathrm{C}$ (Dong, Ruan et al. 2004; Mucha and Pawlak 2005).

Por otro lado, el proceso de fusión resulta evidenciado por un pico endotérmico en la curva DSC (Figura 2). Para el hidrogel con un menor contenido de ácido acético glacial (HQ 0.5) fue de 123 ${ }^{\circ} \mathrm{C}$, para el hidrogel con un porcentaje medio de ácido (HQ 1) de $139{ }^{\circ} \mathrm{C}$, y finalmente, para el hidrogel con mayor contenido de ácido (HQ 2), la temperatura de fusión fue de $142{ }^{\circ} \mathrm{C}$. Estos resultados pudieran estar relacionados a que un mayor contenido de ácido acético, que actúa como entrecruzante en el quitosano, forma un hidrogel más rígido, duro y con un valor de temperatura de fusión mayor (Francisco Gabriele y Col., 2021), es decir, un mayor grado de reticulación requiere una mayor temperatura para fundir la parte cristalina de la matriz de quitosano, resultando en un incremento en la temperatura fusión (Tm), debido al incremento en porcentaje del ácido acético.

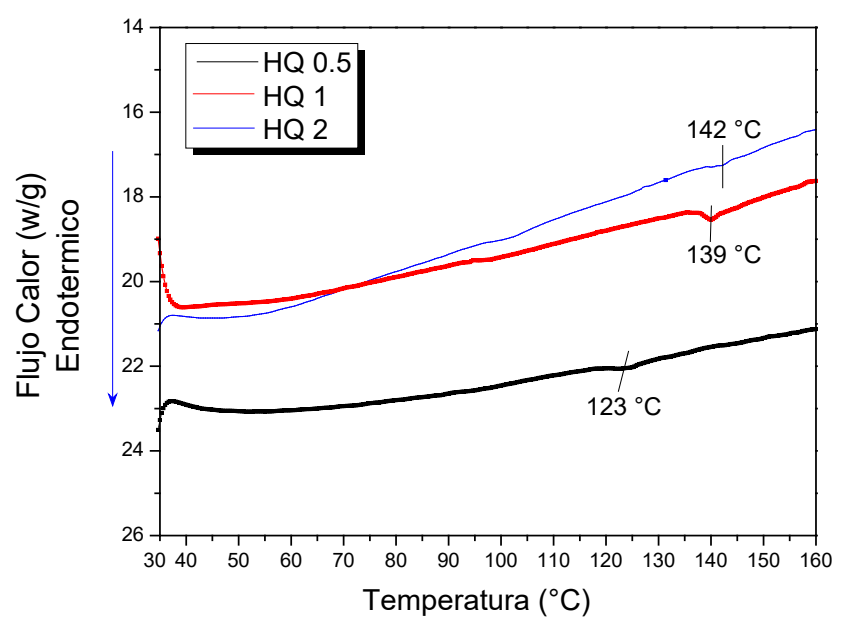

Figura 2. Curva de DSC para los hidrogeles de quitosano con diferentes concentraciones de ácido acético glacial.

La curva de TGA en la Figura 3, indica dos diferentes pérdidas de peso, la primera pérdida de peso se observa en un intervalo de 100 a $200{ }^{\circ} \mathrm{C}$, atribuida al agua físicamente absorbida, debido a que el quitosano es un material higroscópico. A partir de $200{ }^{\circ} \mathrm{C}$, la segunda pérdida de peso corresponde a una pirólisis del biopolímero, iniciando con el rompimiento de los enlaces glucósidos seguida por el proceso de oxidación que corresponde a la desacetilación del quitosano, entre los 450 a $620{ }^{\circ} \mathrm{C}$, y finalmente la degradación del quitosano después de $620{ }^{\circ} \mathrm{C}$ (Bonilla-Cruz, Lara-Ceniceros et al. 2007). En este análisis de TGA se observa una mayor estabilidad térmica para el hidrogel de quitosano, con mayor porcentaje de ácido acético glacial, y que esta estabilidad va disminuyendo conforme la relación del ácido acético glacial es menor en el hidrogel.

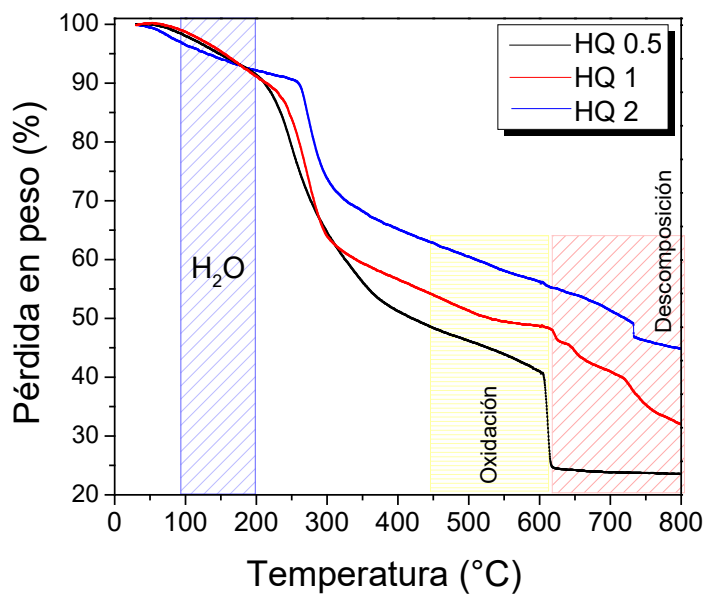

Figura 3. Curva de TGA para los hidrogeles de quitosano con diferentes concentraciones de ácido acético glacial.

Una vez caracterizados los hidrogeles, se cargaron con extracto de romero, para evaluar su biocompatibilidad, mediante la prueba de 
hemólisis. La interacción de los hidrogeles de quitosano cargados con extracto de romero con los componentes sanguíneos puede conducir a la lisis de los eritrocitos humanos. Por esta razón, los efectos de los hidrogeles en la sangre se evaluaron con ensayos de hemólisis (Figura 4). La Norma ASTM F 176-17 indica los siguientes parámetros para medir el porcentaje de hemólisis: $<2$ $\%$ no hemolítico, 2-4 \% ligeramente hemolítico, $>5 \%$ hemolítico. Los resultados obtenidos se consideran no hemolíticos al presentar porcentajes inferiores al $2 \%$ de hemólisis (ASTM, 2018).

Los hidrogeles blanco presentaron un porcentaje de hemólisis del $0.3 \%$, los hidrogeles que contenían extracto de romero presentaban un porcentaje de hemólisis alrededor del 1\%. Para los hidrogeles cargados con extracto de romero y modificados por tecnología de plasma su porcentaje de hemólisis fue de aproximadamente $0.5 \%$. Después del tratamiento con plasma, se observó una disminución en el porcentaje de hemólisis de las muestras, ya que los valores obtenidos eran similares a los obtenidos con el hidrogel blanco, estos resultados confirman que el tratamiento con plasma ayuda a disminuir el efecto nocivo que pueden provocar estas partículas en el torrente sanguíneo al ser utilizados en concentraciones de $1,2.5$ y $5 \mathrm{mg} / \mathrm{mL}$ y avalan que esta tecnología pueda ser utilizada para mejorar la biocompatibilidad de estos materiales. Esto posiblemente gracias a la remoción de contaminantes en la superficie de los materiales $\mathrm{y}$ una posible inserción de grupos funcionales como los grupos amino primarios, que los hacen compatibles con eritrocitos humanos, ya que el tratamiento con plasma se realizó con aire, y es posible tener funcionalidades de este tipo en la superficie.

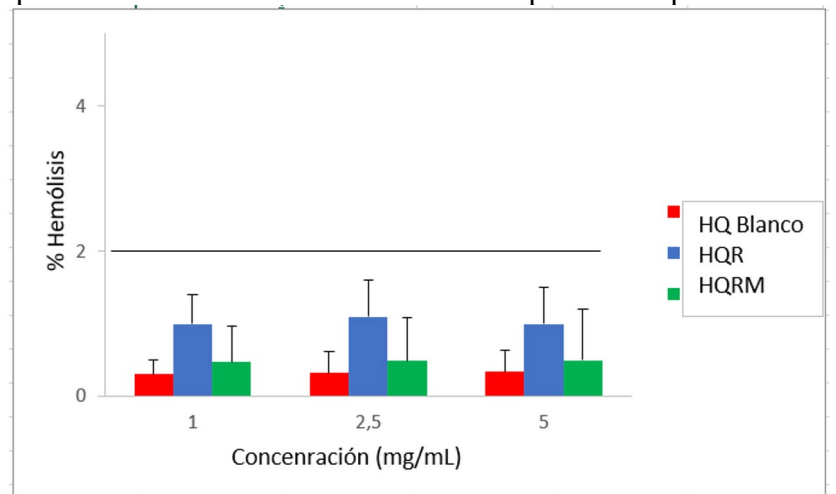

Figura 4. Gráfico de resultados de pruebas de hemólisis en hidrogeles de quitosano.

\section{Conclusiones}

El hidrogel HQ 0.5, visiblemente fue el más rígido en su estructura física, mientras que el hidrogel HQ 2 fue el más manejable, los resultados de IR indican la presencia de quitosano en los hidrogeles. El hidrogel HQ 2 fue el más estable térmicamente, según lo observado en las curvas de DSC y TGA. La tecnología de plasma de radiofrecuencia es una herramienta con la capacidad de modificar superficies para la obtención de compuestos biocompatibles, esto se pudo comprobar en las pruebas de hemólisis ya que los hidrogeles tratados con plasma presentaban una disminución del porcentaje de hemólisis en comparación con los hidrogeles de quitosano cargados con extracto de romero sin tratar.

\section{Agradecimientos}

Los autores agradecen al Consejo Nacional de Ciencia y Tecnología (CONACYT), por la beca otorgada a Claudia Gabriela
Cuéllar Gaona, con número de CVU 618041, para sus estudios doctorales.

\section{Referencias}

Ahmed, M. E., Mohamed, H. M., Mohamed, M. I., Kandile, N. G. (2020). "Sustainable antimicrobial modified chitosan and its nanoparticles hydrogels: Synthesis and characterization." International Journal of Biological Macromolecules 162: 1388-1397.

ASTM. (2018). Standard Practice for Assessment of Hemolytic Properties of Materials 1. 1-6. https://doi.org/10.1520/F0756-17.Copyright

Ben Abada, M., et al. (2020). "Variations in chemotypes patterns of Tunisian Rosmarinus officinalis essential oils and applications for controlling the date moth Ectomyelois ceratoniae (Pyralidae)." South African Journal of Botany 128: 18-27.

Biduski, B., et al. (2019). "Electrosprayed octenyl succinic anhydride starch capsules for rosemary essential oil encapsulation." International Journal of Biological Macromolecules 132: 300-307.

Bonilla-Cruz, J., Lara-Ceniceros, T., Saldívar-Guerra, E., Jiménez-Regalado, E. (2007). "Towards Controlled Graft Polymerization of Poly[styrene-co(maleic anhydride)] on Functionalized Silica Mediated by Oxoaminium Bromide Salt. Facile Synthetic Pathway Using Nitroxide Chemistry." Macromolecular Rapid Communications 28(13): 1397-1403.

Dhand, A. P., Galarraga, J. H., Burdick, J. A. (2020). "Enhancing Biopolymer Hydrogel Functionality through Interpenetrating Networks." Trends in Biotechnology.

Dong, Y., Wang, H., Ruan, Y., Zhao, Y. (2004). "Studies on glass transition temperature of chitosan with four techniques." Journal of Applied Polymer Science 93(4): 1553-1558.

Elmi, A., et al. (2019). "Antimicrobial capabilities of non-spermicidal concentrations of tea tree (Melaleuca alternifolia) and rosemary (Rosmarinus officinalis) essential oils on the liquid phase of refrigerated swine seminal doses." Research in Veterinary Science 127: 76-81.

Forouzandehdel, S., et al. (2020). "Synthesis of a novel magnetic starch-alginic acid-based biomaterial for drug delivery." Carbohydrate Research 487 107889.

Francesco Gabriele., et al.(2021. "Ionic and covalent crosslinking in chitosansuccinic acid membranes: Effect on physicochemical properties." Carbohydrate Polymers 21: 117106.

Karadağ, A. E., et al. (2019). "In vitro antibacterial, antioxidant, antiinflammatory and analgesic evaluation of Rosmarinus officinalis L. flower extract fractions." South African Journal of Botany 125: 214-220.

Liu, Q., Xiong, L., Sun, Q., Ji, N. (2020). "Rapid gelling, self-healing, and fluorescence-responsive chitosan hydrogels formed by dynamic covalent crosslinking." Carbohydrate Polymers 246: 116586.

Lopez-Carrizales, M., et al. (2020). "Characterization, antibiofilm and biocompatibility properties of chitosan hydrogels loaded with silver nanoparticles and ampicillin: an alternative protection to central venous catheters." Colloids and Surfaces B: Biointerfaces 196: 111292.

Loto, R. T. and C. A. Loto (2019). "Data on the comparative evaluation of the corrosion inhibition of vanillin and vanillin admixed with rosmarinus officinalis on mild steel in dilute acid media." Chemical Data Collections 24: 100290.

Lu, T.-Y., Chen, Y., Huang, W. (2020). "Effect of varied hair protein fractions on the gel properties of keratin/chitosan hydrogels for the use in tissue engineering." Colloids and Surfaces B: Biointerfaces 195: 111258.

Maiz-Fernández, S., Guaresti, O., Pérez-Álvarez, L., Ruiz-Rubio, L., Gabilondo, N., Vila-Vilela, J. L., Lanceros-Méndez, S. (2020). " $\beta$-Glycerol phosphate/genipin chitosan hydrogels: A comparative study of their properties and diclofenac delivery." Carbohydrate Polymers 248: 116811.

Mucha, M. and A. Pawlak (2005). "Thermal analysis of chitosan and its blends." Thermochimica Acta 427(1): 69-76.

Rezaei, F. S., Sharifianjazi, F., Esmaeilkhanian, A., Salehi, E. (2021). "Chitosan films and scaffolds for regenerative medicine applications: A review." Carbohydrate Polymers 273: 118631.

Tran, D. L. (2020). "Novel enzymatically crosslinked chitosan hydrogels with free-radical-scavenging property and promoted cellular behaviors under hyperglycemia." Progress in Natural Science: Materials International.

Wei, W., Yuanzhu, M., Xudong, Y., Wenyan, Z., Xiaozhao W., Chenglin, L., Junxin, L., Qiulin, H., Sebastian, L., Hongwei, O. (2021). "Advanced hydrogels for the repair of cartilage defects and regeneration." Bioactive Materials 6(4): 998-1011.

Zhong, X., et al. (2020). "Chemical characterization of the polar antibacterial fraction of the ethanol extract from Rosmarinus officinalis." Food Chemistry: 128674 